\title{
„E pluribus unum“: Zertifizierte Fortbildung Senologische Radiologie feiert RöKo-Premiere
}

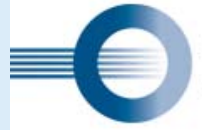

AC Mammadiagnostik

in der Deutschen Röntgengesellschaft

Auf dem 99. Deutschen Röntgenkongress stellt die AG Mammadiagnostik der DRG ein neues Fortbildungszertifikat vor, mit dem Radiologinnen und Radiologen ihr Spezialwissen nachweisbar ausbauen können. Prof. Dr. Markus Müller-Schimpfle, Chefarzt der Klinik für Radiologie und Neuroradiologie, Schwerpunkt Radiologie am Klinikum Frankfurt-Höchst und Vorsitzender der AG, skizziert im Interview die Besonderheiten der Zertifizierung und ihre Genese.

\section{Herr Professor Müller-Schimpfle, wie lässt sich die bildgebende Mammadiagnostik innerhalb des radiologischen Leistungs- portfolios verorten?}

Grundsätzlich ist es ja so, dass die Radiologie ein sehr breit aufgestelltes Fach ist. Wir diagnostizieren und therapieren Patienten in einem großen klinischen Anwendungsspektrum und in allen Altersklassen. Die Mammadiagnostik ist Teil dieser ganzheitlichen medizinischen Versorgung einschließlich ihrer zentralen Rolle im sekundären Präventionsprogramm des MammografieScreenings. Eine Schwerpunktlegung findet in der Regel schon in der Facharztausbildung statt, je nach Weiterbildungsinstitution. Die tiefere fachliche Fokussierung geht dann aber über das in der Weiterbildungsordnung geforderte Wissen hinaus.

Um dieses senologische Spezialwissen zu dokumentieren, hat die AG Mammadiagnostik jetzt ein eigenes Zertifizierungsangebot auf den Weg gebracht. Welche Kompetenzen sollen gefördert werden und wie ist die Zertifizierung aufgebaut?

In der Stufe Q1 geht es im Wesentlichen darum, auf Grundlage der mammadiagnostischen Weiterbildung besondere Kenntnisse inklusive des Mamma-Ultraschalls darzulegen. Beispielsweise werden mindestens 300 Mamma-Ultraschalluntersuchungen gefordert, davon mindestens einhundert pathologische Befunde. Stufe Q2 fordert die Kenntnis aller drei Modalitäten inklusive der MRT. Denn eine Mamma-MRT ist typischerweise etwas, was man im Rahmen der radiologischen Weiterbildung sicherlich mal sieht, aber nicht im Sinne einer strukturierten Kenntnisvermittlung erlernt. Auch die interventionellen Verfahren haben wir erst in Stufe Q2 aufgenommen, denn für diese schwierigen Anwendungen ist einige belegbare Vorerfahrung notwendig. Das Anforderungsprofil für einen Facharzt auf Q2-Niveau umfasst die eigenständige Tätigkeit in einem Zentrum, sei es ein Brustzentrum oder eine Screening-Einheit. Was bei dem Nachweis wichtig zu wissen ist: Die geforderten Zahlen umfassen immer den Dreiklang aus Mammografie, Sonografie und MRT plus interventionellen Elementen. Denn Einzelkenntnisse sehen wir häufig, aber wer seine besondere Expertise in der senologischen Radiologie darstellen möchte, muss das synoptische Lesen der drei Modalitäten beherrschen. Eine Herausforderung - das ist uns bewusst. Aber wir wollen das Zertifikat nicht zu einer Hürde machen, sondern die Qualität der Mammadiagnostik fördern. Denn für alle drei Säulen der Mammadiagnostik gibt es bereits Qualitätssicherungsvereinbarungen, die wir mit Leben füllen wollen. Stufe Q3 schließlich soll diejenigen auszeichnen, die auch in der Fort- und Weiterbildung, also in der Kenntnisvermittlung, tätig sind. Denn wir alle wissen: Etwas selbst zu können ist eine sehr gute Qualifikation, aber etwas vermitteln zu können erfordert typischerweise einen ganz anderen Grad der Selbsterkenntnis und Reflektion über das Fachgebiet. Deswegen war es uns wichtig, diese Supervisionsexpertise mit einer eigenen Stufe zu kennzeichnen.

Wie haben Sie in Vorbereitung auf das Zertifizierungskonzept die Bedarfe der Kollegen ermittelt?

Die Idee, die Mamma-Spezialqualifikation durch eine zertifizierte Fortbildung zu formalisieren, gibt es sicher schon zehn Jahre, immer mit dem Spagat vor Augen: „e pluribus unum“, also in der Vielfalt die Einheit

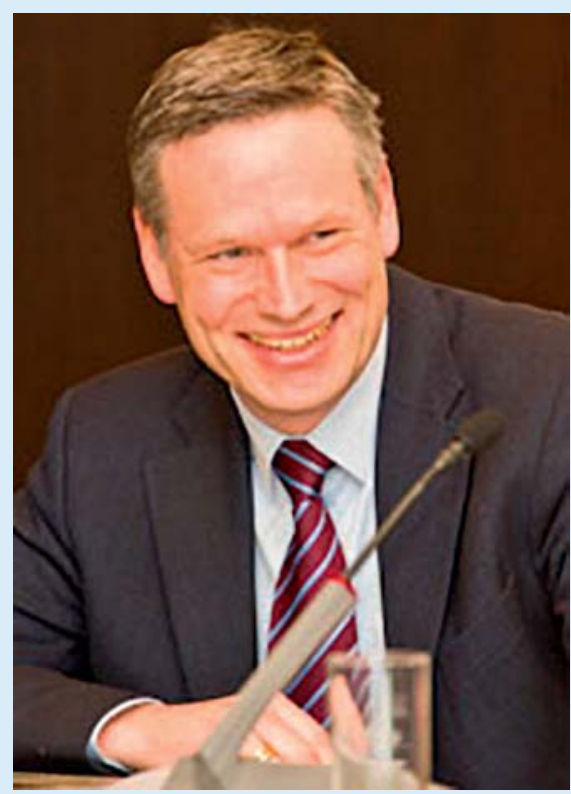

Prof. Dr. Markus Müller-Schimpfle

der Radiologie zu erhalten. Ich glaube, das ist uns mit dieser Zertifikatsfortbildung gut gelungen, denn wie ich schon sagte: Die Radiologie ist ein großes Fach, hat aber auch einzelne Spezialgebiete, die wir ebenso fördern wollen. Im Vorstand haben wir intensiv dazu diskutiert und vor zwei Jahren die Mitglieder dazu befragt. Der einhellige Auftrag: eine zertifizierte Fortbildung zu entwickeln. Die eigentliche Konzeptionsphase ging dann recht flott, innerhalb eines Jahres. Nach Rücksprache mit den AG-Mitgliedern konnten wir uns im zweiten Jahr gemeinsam mit dem DRG-Vorstand dem Feinschliff widmen. Deshalb gehen wir davon aus, dass der Bedarf da ist und die Kollegen sich auf die Zertifizierung freuen, weil sie ihre speziellen Kenntnisse und Fähigkeiten nun darstellen können. Das Schöne ist: Mit diesem Zertifikat lässt sich auch gegenüber Patientinnen kenntlich machen, dass hier Ärzte in der Mammadiagnostik und -intervention arbeiten, die wirklich wissen, wovon sie sprechen. Das gilt für alle Anwender, egal, ob sie in einer Privatpraxis, einer GKV-Praxis, einer Ambulanz oder einem Institut arbeiten. 
Haben Sie eine bestimmte Anzahl an Kursen für das Jahr 2018 geplant?

Grundsätzlich möchte ich betonen, dass es auf den verschiedenen Fachveranstaltungen bereits sehr gute Fortbildungskurse gibt, sowohl beim RöKo, bei Veranstaltungen der Deutschen Gesellschaft für Senologie und der Deutschen Gesellschaft für Ultraschall in der Medizin, oder auch auf internationalen Veranstaltungen wie den Treffen der EUSOBI. Sie alle bieten ein hohes Niveau und sind hilfreich für unsere Zertifikatsfortbildung. Wir legen deshalb den Schwerpunkt auf das Erreichen der CME-Punkte und der passenden Inhalte. Damit wollen wir ganz gezielt die Fortbildung fordern und fördern, aber kein eigenes Format mit möglicherweise problematischen finanziellen Anreizen schaffen.

Sie starten mit dem Zertifizierungsprogramm auf dem 99. RöKo. Bieten Sie dort ganz konkret Veranstaltungen an?

Zunächst einmal wollen wir die AG-Mitglieder präzise informieren, wie genau die Zertifizierung aussieht. Ergänzend haben wir einzelne Veranstaltungen auf dem RöKo gekennzeichnet - sie sind zur Anerkennung im Rahmen des Zertifikats geeignet. Gedacht sind sie allerdings mehr als Teaser im Programm: „Ach, schaut mal, da tut sich ja was!“ Darüber hinaus sind wie gesagt alle anderen Veranstaltungen zur Vorlage für das Zertifikat sehr willkommen, die über die Akademie anerkannt sind und auf dem Gebiet der senologischen Radiologie stattfinden.

Die neue Zertifizierung gilt für einzelne Radiologinnen und Radiologen. Sind weitere Zertifizierungsarten in Planung zum Beispiel für Zentren?

Im Vorstand haben wir diskutiert, ob wir die Zertifizierung auch auf Zentren ausdehnen sollten. Unter dem Strich lässt sich aber sagen, dass die Zentren typischerweise durch Screening-Einheiten auf der einen Seite und Brustzentren auf der anderen Seite in Deutschland bereits auf hohem Niveau struktur-, prozess- und ergebnisorientiert arbeiten. Was uns bislang fehlte, ist in der Tat die persönliche Qualitätsdarstellung. Aktuell sind wir im Gespräch mit der Zertifizierungskommission der Deutschen Krebs- gesellschaft. Denn nun haben wir einen transparenten Zertifizierungsprozess aufgesetzt, von dem wir sicher sind, dass Zentren davon immens profitieren, besonders durch die Kombination der drei Modalitäten auf den Stufen Q2 und Q3.

Gibt es Übergangsregelungen für die Stufen Q2 und Q3?

Ja, die gibt es - bis zum RöKo 2020 ist der Direkteinstieg auf den Qualifizierungsstufen Q2 bzw. Q3 möglich. Schließlich wollen wir Ärzten, die schon weit fortgeschritten sind, keine sinnlosen Hürden in den Weg stellen. Wir sind angewiesen auf diejenigen, die bereits auf einer der drei Stufen arbeiten. Natürlich insbesondere auf die Q3-Experten, die dann als Referenten überhaupt erst die Voraussetzung schaffen, dass die senologische Radiologie gelehrt wird. Anträge auf allen drei Stufen sollten durch anonymisierte Auszüge aus dem Radiologie-Informationssystem belegt werden. Außerdem gilt, dass für Anträge, die bis zum 31.12.2018 in der DRG-Geschäftsstelle eingehen, keine Zertifizierungsgebühren erhoben werden. 